\title{
Identification of Brassica oleracea monosomic alien chromosome addition lines with molecular markers reveals extensive gene duplication
}

\author{
J. Mitchell McGrath ${ }^{1, *}$, Carlos F. Quiros ${ }^{1}$, John J. Harada ${ }^{2}$, and Benoit S. Landry ${ }^{3}$ \\ ${ }^{1}$ Department of Vegetable Crops, University of California, Davis, CA 95616, USA \\ 2 Department of Botany, University of California, Davis, CA 95616, USA \\ ${ }^{3}$ Agriculture Canada Research Center, P.O. Box 457, St-Jean, Quebec, Canada, J3B 6Z8
}

Received March 16, 1990

Summary. Chromosomes of Brassica oleracea $(2 n=18)$ were dissected from the resynthesized amphidiploid $B$. napus Hakuran by repeated backcrosses to $B$. campestris $(2 \mathrm{n}=20)$, creating a series of monosomic alien chromosome addition line plants $(2 n=21)$. Using morphological, isozyme and restriction fragment length polymorphism markers (RFLPs), 81 putative loci were identified. Of nine possible synteny groups, seven were represented in the 25 monosomic addition plants tested. Sequences homologous to $26 \%$ of the 61 DNA clones utilized ( $80 \%$ were cDNA clones) were found on more than one synteny group, indicating a high level of gene duplication. Anomalous synteny associations were detected in four $2 n=21$ plants. One of these plants showed two markers from one $B$. oleracea chromosome associated with a second complete $B$. oleracea synteny group, suggesting translocation or recombination between non-homologous chromosomes in Hakuran or the backcross derivatives. The other three $2 n=21$ plants each contained two or more $B$. oleracea synteny groups, suggesting chromosome substitution.

Key words: Restriction fragment length polymorphism - Chromosome substitution - Non-homologous recombination - Genetic stocks - Aneuploidy

\section{Introduction}

The genus Brassica is characterized at the diploid level by chromosome numbers ranging from $n=7$ to $n=11$ (Gomez-Campo and Hinata 1980). However, from karyotypes of pachytene chromosomes (Robbelen 1960) and chromosome pairing affinities in haploids of $B$. oleracea (Armstrong and Keller 1982) and B. campestris (Armstrong and Keller 1981), the primary chromosome

\footnotetext{
* Present address: Department of Biology, University of Michigan, Ann Arbor, MI 48109-1048, USA

Offprint requests to: J.M.McGrath
}

number in this genus is presumed to be $x=6$. The suggestion that current Brassica species are secondary polyploids, with the implication that extant Brassica species have evolved by aneuploid increase from an extinct ancestor, was first put forward by Catcheside (1937).

Along with an aneuploid series at the diploid level, Brassica also exhibits a similar series at the tetraploid level. Genome analysis by Morinaga (1934) and U (1935) showed the polyploid series to be amphidiploid combinations of diploid species, a relation widely known as the triangle of $U$. The triangle of $U$ has been confirmed and extended in numerous studies, including those using chloroplast DNA restriction patterns (Palmer et al. 1983) and nuclear DNA restriction fragment length polymorphisms (RFLPs) (Song et al. 1988; Hosaka et al. 1990). However, affinities between diploid species are not as well understood, due to the paucity of available genetic markers and the difficult cytology of Brassica chromosomes.

We are exploiting the ability to hybridize some combinations of diploids and amphidiploids in order to dissect the diploid genomes (Quiros et al. 1987; Hosaka et al. 1990; McGrath and Quiros 1990). The resulting monosomic alien addition lines are useful for the rapid assignment of genes to chromosomes. Our interest lies in comparing gene synteny between monosomic addition lines derived from various amphidiploid species for evolutionary changes associated with aneuploid increase or decrease. Also, we are interested in comparing gene synteny of diploid genomes derived from natural and resynthesized amphidiploids of the same species, to understand process influencing the stability of one genome when combined with another in a common nucleus. Comparison of these syntenies with genetic maps of the natural diploid species will provide a foundation for these comparisons.

Locating genes to chromosomes can be facilitated by the use of aneuploids. With monosomic lines of maize (Helentjaris et al. 1986) and using tomato primary trisomics (Young et al. 1987), rapid and efficient location of cloned DNA fragments to chromosomes has been 
accomplished. Identification of individual $B$. oleracea chromosomes with DNA markers provides a solution to the problems associated with the dearth of morphological and cytological chromosome landmarks exhibited by $B$. oleracea alien monsomic addition lines (McGrath and Quiros 1990).

\section{Materials and methods}

A series of monosomic $B$. oleracea $(2 n=2 x=18$, C-genome designation) chromosome addition lines derived from the artificial B. napus $(2 \mathrm{n}=4 \mathrm{x}=38, \mathrm{AC}$-genome $)$ Hakuran (cabbage $\times$ Chinese cabbage, Nishi 1980) has been constructed in the genetic background of $B$. campestris $(2 \mathrm{n}=2 \mathrm{x}=20$, A-genome $)$ ssp. parachinensis var. Kwan Hoo Choi, and was generated by successively backcrossing Hakuran with $B$. campestris for three generations (McGrath and Quiros 1990). Monosomic addition lines $(2 n+1=20+1)$ were identified by chromosome counts of $80 \mathrm{BC}_{3}$ progeny derived from 27 different $\mathrm{BC}_{2}$ hyperploid plants, generally with one to three extra chromosomes. Twenty-five monosomic addition plants, plus two diploid siblings, were chosen in order to identify $B$. oleracea syntenic groups with RFLP markers. These 25 monosomic addition plants were chosen on the basis of morphology (puckered leaf phenotype, retarded growth), diagnostic isozymes (6Pgd-1, $P g m-1)$ and different $\mathrm{BC}_{2}$ parents, with the goal of recovering all nine $B$. oleracea chromosomes. Methods of chromosome examination, fertility determination and isozyme analyses were those of Quiros et al. (1987). Methods of DNA analysis and the detection of RFLPs are those of McGrath (1989) and Hosaka et al. (1990).

Identification of C-genome specific RFLPs proceeded in two stages. First, polymorphism between the $B$. campestris A-genome and Hakuran was established, and compared to the $B$. olerace $\mathrm{C}$-genome. Probes identifying DNA fragments of the same size in cabbage $(B$. oleracea ssp. capitata) and Hakuran, but not B. campestris, were then used to probe a panel of 25 monosomic addition lines. While the original parents of Hakuran were unavailable, the co-occurrence of a DNA fragment in all accessions with a C-genome was the primary criterion for C-genome specificity and acceptance of the assignment of a marker to a particular addition line. Chromosomes were identified by the complement of $B$. oleracea RFLP markers in a particular plant, and are numbered arbitrarily but consistently with the nomenclature initiated by Quiros et al. (1987).

The majority of probes were prepared from a library of B. napus embryo cDNA clones. A number of these have been shown to be expressed in a developmentally regulated manner (prefaced in Table 1 by $p C O T, p C A$, $p L E A, p A X$, and $p b s$ ) (Harada et al. 1988; Comai et al. 1989a, b; Dietrich et al. 1989). Some anonymous clones (prefixed by $p 1$ and $p 2$ ) were selected on the basis of intergenomic polymorphisms from the same cDNA library (B. Landry, unpublished), and the remaining anonymous cDNA clones (prefixed $p B N$ ) were isolated from the same cDNA library by S. Kianian (Dept. of Vegeta- ble Crops, University of California, Davis). Genomic clones (prefixed $p B$ and $p Y L C$ ) from $B$. oleracea and $B$. napus were from Hosaka et al. (1990). Other clones mapped included an Arabidopsis thaliana alcohol dehydrogenase clone ( $k$ At3011, Chang and Meyerowitz 1986), a chlorophyll A/B binding protein (CAB gene) from tomato $(p L 8, \mathrm{~S}$. Tanksley, personal communication), and $B$. napus napin ( $p N_{2}$, Crouch et al. 1983) and cruciferin $\left(p C_{1}\right.$, Simon et al. 1985) seed storage protein genes. Probes for ribosomal RNA genes (rDNA) included $p T A 71$, containing the entire repeating unit from wheat (Gerlach and Bedbrook 1979), and the intergenic non-transcribed spacer region (D12-3) from radish (Delcasso-Tremousaygue et al. 1988).

\section{Results}

Recovery and identification of monosomic addition line chromosomes

Alien chromosomes were identified with a suite of morphological, isozyme and RFLP markers, arranged without reference to gene order in several syntenic gene associations. One morphological marker (puckered leaf, symbolized PLF), three isozymes (6Pgd-1,Pgm-1 and Got-5) and 61 DNA probes (8 genomic, 49 cDNA, 2 rDNA and 2 heterologous probes) were utilized as genetic markers (Table 1). These markers were distributed more or less evenly across all chromosomes as each synteny group had between 6 and 16 markers. From 134 clones tested for polymorphism between $B$. campestris and Hakuran and conservation of fragments between $B$. oleracea and Hakuran, a total of 74 putative loci were mapped to seven synteny groups recovered in monosomic alien addition lines. An additional seven Cgenome specific bands were tentatively assigned to the two $B$. oleracea synteny groups not recovered.

For most clones tested, analysis was complicated by hybridization patterns characteristic of duplicate genes and gene families. Only 27 of the 134 clones $(20 \%)$ tested for polymorphism between $B$. oleracea and $B$. campestris showed a single band indicative of single copy genes. Virtually all probes $(97.6 \%)$ detected at least one polymorphic fragment between B. oleracea and B. campestris. Within diploid species, $B$. campestris was more polymorphic $(69.4 \%$ of probes detected at least one polymorphism) than was $B$. oleracea (data not shown). Since the C-genome of Hakuran was contributed by an unknown cabbage variety, comparison of B. oleracea fragments represented in Hakuran was a measure of polymorphism within the $B$. oleracea cabbage group $(55.3 \%)$. These values agree with the levels of DNA polymorphism in Brassica determined by Figdore et al. (1988).

Each chromosome contained a number of sequences which were duplicated on other chromosomes. Indeed, duplication of mapped RFLP markers was common. Sixteen of the 61 clones ( $26 \%$ of clones) hybridized to two separate synteny groups, i.e. they were physically mapped to different chromosomes. Most clones recog- 
Table 1. Markers assigned to Brassica oleracea chromosomes

\begin{tabular}{|c|c|c|c|}
\hline \\
\hline & \multicolumn{3}{|c|}{ Synteny group ${ }^{\mathrm{a}}$} \\
\hline$p$ COT44-2 & $p T A 71^{\mathrm{d}}$ & pB850-1 & $p B N 127-1$ \\
\hline pB370-2 & pbsMS1-1 ${ }^{\mathrm{d}}$ & $\overline{\overline{p T A 71}}$ & $\overline{p L 8}=$ \\
\hline$\overline{p B N 127-2}$ & $\overline{p B N 109^{\mathrm{d}}}$ & $\overline{\overline{D 12-3-2}}$ & $\overline{p B N} 63-1$ \\
\hline$\overline{p \overline{p C_{1}}}$ & $p B N 111^{\mathrm{d}}$ & $\overline{p B N 7-1}$ & $\overline{p b s I L 9-1}$ \\
\hline$p 1 N F 6^{\mathrm{c}}$ & $p B N 10$ & $p B 185$ & $p B 547$ \\
\hline$p L E A 76$ & $p B N 120$ & $p B N 5$ & $p B N 25$ \\
\hline$p N_{2}$ & $k A t 3011$ & $p Y L C 24$ & $\begin{array}{l}p B N 79 \\
p B N 113\end{array}$ \\
\hline 5 & 6 & 7 & 8 or $9^{\mathrm{b}}$ \\
\hline$P L F^{\mathrm{g}}$ & $P g m-1$ & $p A X 58-1$ & $p A X 58-2$ \\
\hline $6 P g d-1$ & $p 2 B N 3$ & $\overline{p B N 122-2^{\mathrm{f}}}$ & pB845-2 \\
\hline Got-5 & $\overline{p B 370-1}$ & $\overline{p \text { COT } 44-1}^{\mathrm{a}}$ & $\underline{\overline{p B 850-2}}$ \\
\hline$p 2 B N 3$ & $\overline{p B N 122}-1$ & 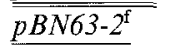 & $\overline{p C A 3-1}$ \\
\hline$p C A 3-2$ & $p T A 71$ & pbsIL9-2 & $\overline{p b s M S t}-2$ \\
\hline$\overline{p L 8}=$ & $D 12-3-1$ & $\overline{p B N 11^{\mathrm{d}}}$ & $\overline{p B N 66}$ \\
\hline$p B N 128-2$ & $p B N 7-2$ & $p B N 69$ & $p Y L C 2$ \\
\hline$p B N 129-2$ & $\overline{p B N 128}-1$ & $p B N 99$ & \\
\hline p1NF & pBN129-1 & $p B N 116$ & \\
\hline p1NF10 & p1NF9 & $p B N 130^{\mathrm{f}}$ & \\
\hline$p B N 6$ & p2NA9 & $p B N 142^{\mathrm{f}}$ & \\
\hline$p B N 14$ & $p B N 55$ & $p C A 12$ & \\
\hline$p B N 33$ & $p B N 12^{e}$ & $p Y L C 5$ & \\
\hline$p B N 46$ & $p B N 57^{\mathrm{e}}$ & $p Y L C 19$ & \\
\hline$p B N 136$ & $p B N 98$ & & \\
\hline$p$ СOT46 & $p B N 121$ & & \\
\hline & $p B N 157$ & & \\
\hline
\end{tabular}

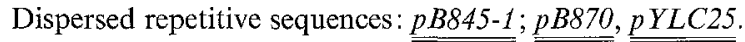

When a particular probe hybridized to fragments on different synteny groups, and recognized different sized fragments, each was assigned a number to identify a unique locus (e.g. $p B N 128-1$ and $p B N 128-2$ ). If sequences were present on more than one synteny group (as defined by association with dissimilar markers), but their fragment size was indistinguishable, no locus designations were made (as with $p T A 71$ and $p L 8$ ). Both $p T A 71$ and $D 12-3$ recognize ribosomal genes

Underline, duplicated synteny; double underline, duplicated loci

${ }^{a}$ Order of markers is arbitrary

b These groups were not recovered as monosomic addition lines

c Deleted in chromosome 1 (plant 603-9)

d Low intensity band scored

e Translocated to chromosome 7 (plant 591-2)

f Deleted in chromosome 7 (plant 603-9)

g 'Puckered leaf' phenotype

nized different sized fragments on different synteny groups, but three ( $p T A 71, p L 8$ and $p 2 B N 3$ ) showed fragments of the same size on different synteny groups as identified by different syntenic marker associations. In addition to a strong signal on two $B$. oleracea synteny groups, clone $p T A 71$ hybridized weakly to a third. Of the 81 putative loci, 34 mapped to two or three synteny groups ( $42 \%$ of loci) (Table 2 ). This represents an underestimate, since some fragments were not polymorphic between $B$. olerace $a$ and $B$. campestris using the restriction endonuclease EcoRI, and thus could not be
Table 2. Number of markers per chromosome and the proportion of these markers duplicated to any other synteny group

\begin{tabular}{lccll}
\hline $\begin{array}{l}\text { Chromo- } \\
\text { some }\end{array}$ & $\begin{array}{l}\text { Total } \\
\text { loci }\end{array}$ & $\begin{array}{l}\text { Percentage } \\
\text { of } \\
\text { total }\end{array}$ & $\begin{array}{l}\text { Duplicate } \\
\text { loci }\end{array}$ & $\begin{array}{l}\text { Proportion } \\
\text { duplicated } \\
(\%)\end{array}$ \\
\hline 1 & 7 & 8.6 & 3 & 42.9 \\
2 & 7 & 8.6 & 2 & 28.6 \\
3 & 6 & 7.4 & 3 & 50.0 \\
4 & 8 & 9.9 & 4 & 50.0 \\
5 & 16 & 19.8 & 5 & 31.2 \\
6 & 16 & 19.8 & 7 & 43.8 \\
7 & 14 & 17.3 & 5 & 35.7 \\
8 and 9 & 7 & 8.6 & 5 & 71.4 \\
\hline Total & 81 & 100.0 & 34 & 42.0 \\
\hline
\end{tabular}

Dispersed repeat sequences were not included in this table

mapped. For instance, both napin and cruciferin belong to gene families (Crouch et al. 1983; Simon et al. 1985), but only one locus for each was detected in this analysis. Duplicated loci were not confined to a subset of chromosomes, but were found in similar proportions on all recovered synteny groups (Table 2). All duplicated loci detected here are unlinked. Duplicated loci located on the same synteny group would not be identified using these procedures.

Sequences dispersed to three or more synteny groups were identified with genomic clones $p B 845-1, p B 870$, and $p Y L C 25$. All plants positive for a specific marker had fragments of identical size. Unexpectedly, however, these dispersed repetitive sequences were not detected in all plants carrying a particular chromosome. Probe $p$ B845-1 was present in the single plant carrying chromosome 3 , one of two plants carrying chromosome 2 , and 10 of 16 plants carrying chromosome 7 . However, the same probe revealed a second locus ( $p B 845-2)$ which may be located on a single chromosome (chromosome 8 or 9 ). Probe $p Y L C 25$ was found in all plants with chromosomes 2 and 6 , and in 6 of 16 lines carrying chromosome 7. Sequences hybridizing with probe $p B 870$ were scattered among chromosomes: of two plants with chromosome 1 , one carried this sequence. This sequence was also detected on two of three plants with chromosome 6 , each of two chromosome 2 and 5 plants, and 15 of 16 lines carrying chromosome 7 .

Several locus pairs were found distributed among chromosomes (Table 1). A pair of loci ( $p$ BN128-1 and $p B N 129-1$ ) located on chromosome 6 are also present on chromosome 5 ( $p B N 128-2$ and $p B N 129-2)$. Another pair of loci, also on chromosome $6(r D N A$ and $p B N 7-2)$, are duplicated on chromosome 3 (rDNA and $p B N 7-1$ ). Thus, four loci syntenic on chromosome 6 were apparently duplicated and divided between two other synteny groups, chromosomes 3 and 5 . Another pair of syntenic loci ( $p b s I L 9$ and $p B N 63$ ) were located to chromosomes 4 and 7 . The remaining nine duplicated locus pairs were distributed on different chromosomes. 
1234

A

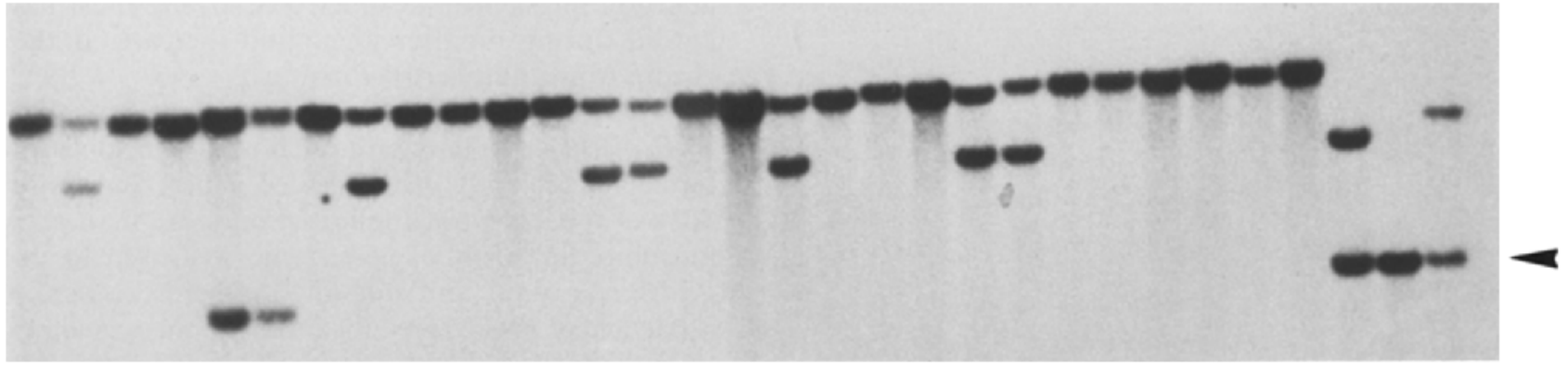

B

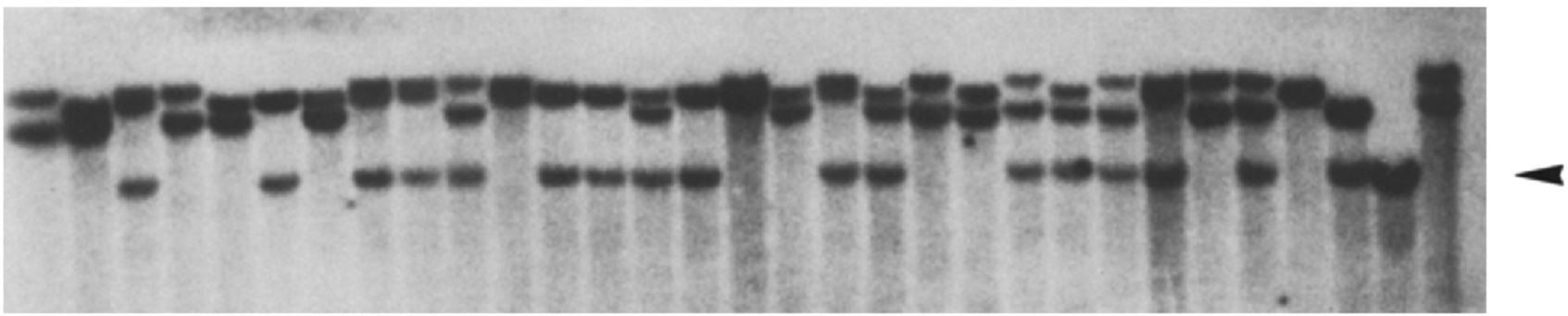

C

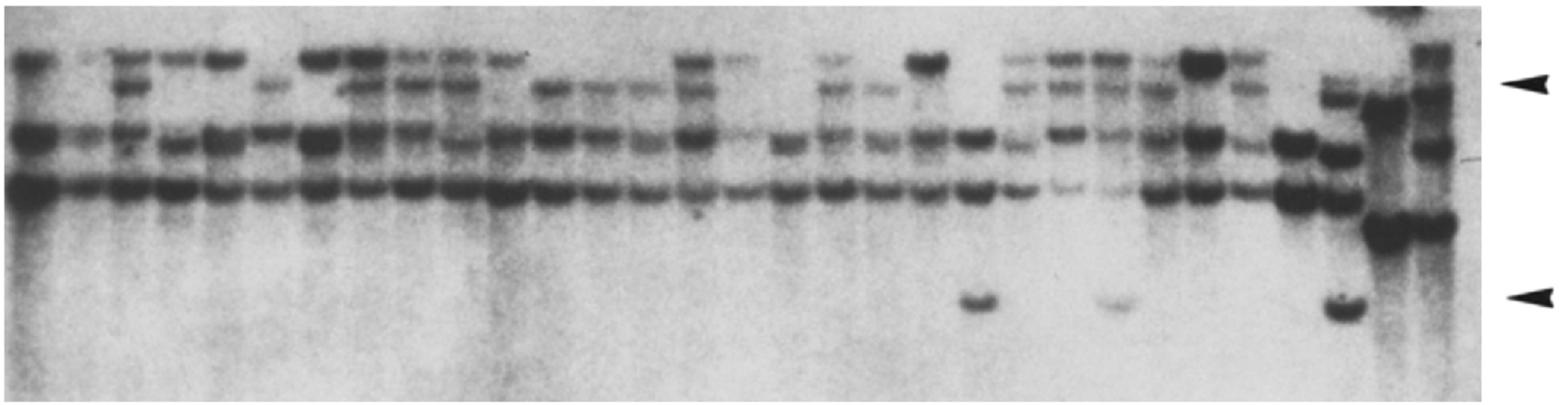

Fig. 1. Sample monosomic addition line blots. 1. Brassica campestris ssp. parachinensis cv. Kwan Hoo Choi (Accession B233 obtained from Redwood City Seed, Redwood City, Calif.). 2. B. napus cv. Hakuran (Accession B454, International Vegetable Research and Development Center, Taiwan). 3. B. oleracea spp. capitata cv. Early Jersy Wakefield (Accession B52, Northrup King, Gilroy,
Calif.). 4. B. napus, Rapeseed (Accession B482, Johnny's Selected Seeds, Albion, Me.). All other lanes are addition lines. A. Probe $p B N 33$ recognizes synteny group 5 (two plants, arrowhead). B. Probe $p C A 12$ is located to synteny group 7 . Notice the high frequency of this marker (arrowhead). C. Probe pbsIL9 maps to at least two synteny groups, chromosomes 4 and 7 (arrowhead)

\section{Anomalous chromosome associations}

Most synteny groups were isolated in duplicate or triplicate from independent $\mathrm{BC}_{2}$ hyperploid plants. In particular, synteny groups 1 and 6 were each recovered in three plants, and synteny groups 2,4 and 5 were each found in two plants. However, both chromosome 2 monosomic addition lines were derived from the same $\mathrm{BC}_{2}$ plant. Only one plant carrying $B$. oleracea chromosome 3 was recovered. Sixteen plants, more than half of the 25 monosomic addition lines, carried chromosome 7. Not until a number of markers were mapped was it clear that these were not segregating $B$. campestris markers (Fig. 1). No unusual syntenic associations were identified in the two diploid $(2 \mathrm{n}=20)$ siblings analyzed, and all markers scored in these individuals could be assigned to B. campestris.

In general, all plants which carried a particular chromosome showed the same syntenic marker associations, indicating the stability of alien chromosomes during con- struction of these lines. Monosomic addition lines were identified by the presence of 21 staining bodies at meiotic metaphase II, or by 11 staining bodies at diakinesis. Most lines with 11 staining bodies at diakinesis had predominantly 10 bivalents plus 1 univalent. However, cytological identification of individual $B$. oleracea chromosomes was rarely possible. The first indication of anomalous patterns detected in this population of 25 monosomic addition lines was that molecular markers identified 29 synteny groups.

Plants 589-5 and 600-1 (each $2 \mathrm{n}=21$ ) were examined at both metaphase II and at diakinesis, and typically one univalent and ten bivalents were visible. However, RFLP markers indicated two entire $B$. oleracea synteny groups were carried by these plants (Table 3 ). These results were suggestive of chromosome substitution. Another $2 n=21$ plant (591-2) was recovered which contained, in addition to an entire intact synteny group 7 , two additional markers derived from synteny group 6 (Table 1). Recombination between non-homologous $B$. 
Table 3. Fertility of $2 \mathrm{n}=21 \mathrm{BC}_{3}$ plants with substituted chromosomes

\begin{tabular}{llll}
\hline Plant & Chromosome & $\begin{array}{l}\text { Male } \\
\text { fertility (\%) }\end{array}$ & $\begin{array}{l}\text { Female } \\
\text { fertility (\%) }\end{array}$ \\
\hline $589-5$ & 6,7 & 50.1 & 0.0 \\
$591-2$ & $7^{\mathrm{a}}$ & 44.8 & 1.3 \\
$600-1$ & 4,7 & 53.0 & $6.3^{\mathrm{c}}$ \\
$603-9$ & $1^{\mathrm{b}}, 5,7^{\mathrm{b}}$ & 0.0 & 0.0 \\
$2 \mathrm{n}=21$ average & & 67.0 & 46.2 \\
\hline
\end{tabular}

a Contains markers from chromosome 6

b Partial synteny groups (see Table 1)

${ }^{c}$ Indicates backcross data; all others are self data

oleracea chromosomes or between $B$. oleracea and $B$. campestris chromosomes would explain this result. A fourth monosomic addition plant (603-9) carried an entire synteny group 5 and nearly intact synteny groups from both chromosomes 1 and 7 . Extensive multivalent formation rendered it difficult to determine chromosome number at diakinesis, but this plant contained 21 chromosomes at metaphase II. Plants with altered synteny associations showed reduced fertility relative to other monosomic addition lines (Table 3 ). In the three plants where substitutions were detected, chromosome 7 was always involved.

\section{Discussion}

Seven of nine possible $B$. oleracea synteny groups were recovered as monosomic addition lines. Additional $\mathrm{C}$ genome specific markers were also identified through their hybridization with control plants which carried a C-genome (i.e. B. oleracea, B. napus and Hakuran), but did not hybridize to any monosomic addition line. These C-genome markers presumably identify one or both of the two syntenic groups which remain to be recovered (i.e. synteny groups 8 and 9 ). One of these unmapped C-genome specific markers ( $p$ B850-2 tentatively assigned to chromosome 8 ) was present in 6 of $26 \mathrm{BC}_{2}$ hyperploid plants, but absent in all monosomic addition lines recovered. Marker pB850-2 and other unmapped C-genome specific markers will facilitate the recovery of the remaining two synteny groups as monosomic addition lines in future generations.

Molecular markers currently provide the best means to identify Brassica chromosomes. The lack of cytological and genetic markers has precluded development of a standard nomenclature for Brassica chromosomes. Six types of chromosomes were described by Robbelen (1960) on the basis of centromere position and heterochromatic knobs and suggested that three types were present in duplicate in the $B$. oleracea genome. Although there is no reason a priori, for assuming Brassica genomes have evolved by simple aneuploid increase, it presents a simple explanation for the observed range of chromosome numbers. As there are at least seven different $B$. oleracea synteny groups represented in these addition lines, chromosome repatterning must have occurred during or after aneuploid increase, if the base chromosome number of Brassica is $\mathrm{x}=6$.

The majority of clones (primarily cDNA) localized to $B$. oleracea synteny groups showed hybridization patterns characteristic of duplicated genes, and more than $40 \%$ of the loci were duplicated on more than one chromosome. Since only one enzyme was used to uncover RFLP variation and not all fragments recognized by a particular probe were localized to chromosomes, our estimates of gene duplication in $B$. oleracea are conservative. Using the same clones we have mapped a similar proportion of duplicated genes in an $F_{2}$ population of B. campestris (McGrath 1989). Through mapping 135 low copy number genomic sequences on segregating populations of both $B$. oleracea and $B$. campestris, Slocum (1989) identified duplicate genes with $35 \%$ of the clones tested. Conversely, the proportion of single copy genes localized to chromosomes is very low, in this study $12 \%$ of the 49 cDNA clones utilized were single copy, and Slocum (1989) identified 7\% of mapped low copy number genomic sequences as single copy.

Three pairs of duplicated syntenic loci homologous to expressed sequences were located in this study. One pair were duplicated on chromosomes 5 and $6(p B N 128$ and $p B N 129)$, another pair mapped to chromosomes 3 and 6 ( $p$ TA71 and $p B N 7$ ), and the third pair to chromosomes 4 and 7 ( $p b s I L 9$ and $p B N 63$ ). The remaining duplicated loci were distributed on various chromosomes. While these loci have not yet been shown to be genetically linked, the observed pattern suggests that in addition to duplication of chromosomes or chromosome segments, rearrangements have occurred in the evolution of the C-genome. In both B. oleracea (Slocum 1989) and maize (Helentjaris et al. 1988) linkage blocks are duplicated between chromosomes but whole chromosome duplication was not evident.

Of the duplicated loci, genes coding for rDNA $(p T A 71)$ and $\mathrm{CAB}$ proteins $(p L 8)$ were found on two or three independent synteny groups, but fragment sizes were conserved. This is suggestive of maintenance of gene structure through some functional constraint, or otherwise mutations which occur at these loci are "selfcorrecting' through some mechanism of concerted evolution. Concerted evolution through selection has been suggested in maintaining gene structure of small subunit ribulose-1,5-bisphosphate carboxylase/oxygenase genes in tomato (Pichersky et al. 1986). However, between two $B$. oleracea syntenic groups derived from Hakuran, rDNA spacer regions show fragment length variation (McGrath 1989). We have also observed two synteny groups carrying rDNA genes in monosomic addition lines derived from natural $B$. napus, but spacer lengths were homogeneous (C.F. Quiros, unpublished). Interchromosome spacer length heterogeneity in Hakuran suggests divergence of these sequences, assuming a common origin of rDNA loci. Also, the detection of weak rDNA hybridization on a third synteny group (group 2), deserves further investigation since it suggests that this chromosome may have relic rDNA sequences. 
Translocation, recombination and chromosome substitution

Three genomic clones were each distributed among three to five alien addition chromosomes, suggesting these are dispersed repetitive elements. It is curious that not all isolates of a particular synteny group contain these sequences, and that each probe recognized an invariant fragment size. This suggests recent changes in chromosome position.

A number of unusual cytological features in these monosomic addition lines were observed, such as multivalent-like associations and disturbed chromosome segregation during meiosis (McGrath and Quiros 1990). This suggests that non-homologous recombination between $B$. oleracea and $B$. campestris chromosomes has an opportunity to occur. Evidence for intergenomic recombination between $B$. oleracea and $B$. campestris chromosomes has been obtained in the transfer of clubroot resistance from $B$. napus to $B$. oleracea (Chiang and Crete 1983) and in the generation of addition lines developed from natural B. napus (Quiros et al. 1987). In this study, one plant (591-2) was recovered with $B$. oleracea markers transferred between chromosomes 6 and 7 , or between chromosome 6 and the $B$. campestris genome. Further breeding of this plant will allow us to distinguish between these possibilities. Non-homologous recombination between unpaired $B$. oleracea chromosomes in hyperploid addition lines may be expected given the level of gene duplication and the probable secondary polyploid nature of its genome.

It is possible that other undetected changes in synteny groups have occurred during the initial construction of Hakuran or its subsequent breeding, or during the generation of these addition lines. Some unusual results, particularly the distribution of repetitive sequences to some lines but not others carrying the same synteny group, may be explained by recombination between nonhomologous chromosomes within $B$. oleracea and between $B$. oleracea and $B$. campestris. To identify intergenomic recombinants will require analysis of more diploid progeny of monosomic addition lines, as B. oleracea chromosome markers were not detected in the two diploid siblings tested here.

Evidence that chromosome substitution occurred was obtained. In three cases, plants with 21 chromatin bodies at metaphase II were demonstrated to contain more than one $B$. oleracea synteny group. It is unusual that each of these plants had $B$. oleracea chromosome 7 in common. Transmission of chromosome 7 was also exceptionally high in this population of monosomic plants. These observations may be related; perhaps this chromosome is functionally equivalent to its $B$. campestris homeolog. That chromosome 7 is not fully equivalent is suggested by the observation that chromosome 7 significantly depressed female fertility in hyperploids (McGrath and Quiros 1990) as well as monosomic addition lines.

Considering the duplicated nature of the $B$. oleracea genomes, substitution lines and nullisomics may be recovered. In the practical generation of alien monosomic chromosome addition lines, however, the frequency of deletions and translocations of $10 \%$ or less observed here does not preclude the recovery of intact $B$. oleracea chromosomes. Rather, the transfer of desirable agronomic traits between these species may be expected at a reasonable rate, and be exploited more fully in Brassica improvement programs. These data also suggest that rare natural interspecific hybridizations have a potential for intergenomic gene transfer, and may be an important mechanism in subspecies differentiation within the crucifer family.

Acknowledgements. We are indebted to E. Meyerowitz, S. Tanksley, K. Hosaka, M. Crouch, M. Saghai-Maroof and M. Delseny for their generous gift of probes. We are also grateful to S. Kianian and V. D'Antonio for technical assistance. This work was supported by USDA competitive grants 86CRCR-1-1926 and 88$37262-4018$ to C.F. Quiros.

\section{References}

Armstrong KC, Keller WA (1981) Chromosome pairing in haploids of Brassica campestris. Theor Appl Genet 59:49-52

Armstrong KC, Keller WA (1982) Chromosome pairing in haploids of Brassica oleracea. Can J Genet Cytol 24:735-739

Catcheside DG (1937) Secondary pairing in Brassica oleracea. Cytologia Jubilee:366-378

Chang C, Meyerowitz EM (1986) Molecular cloning and DNA sequence of the Arabidopsis thatiana alcohol dehydrogenase gene. Proc Natl Acad Sci USA 83:1408-1412

Chiang MS, Crete R (1983) Transfer of resistance to race 2 of Plasmodiophora brassicae from Brassica napus to cabbage ( $B$. oleracea ssp. capitata). V. The inheritance of resistance. Euphytica 32:479-483

Comai L, Baden CS, Harada JJ (1989a) Deduced sequence of a malata synthase polypeptide encoded by a subclass of the gene family. J Biol Chem 264:2778-2782

Comai L, Dietrich RA, Maslyar DJ, Baden CS, Harada JJ (1989 b) Coordinate expression of transcriptionally regulated isocitrate lyase and malate synthase genes in Brassica napus L. The Plant Cell 1:293-300

Crouch ML, Tenbarge KM, Simon AE, Ferl R (1983) cDNA clones from Brassica napus seed storage proteins: evidence from nucleotide sequence analysis that both subunits of napin are cleaved from a precursor polypeptide. J Mol Appl Genet 2:273-283

Delcasso-Tremousaygue D, Grellet F, Panabieres F, Ananiev ED, Delseny M (1988) Structural and transcriptional characterization of the external spacer of a ribosomal RNA nuclear gene from a higher plant. Eur J Biochem 172:767-776

Dietrich RA, Maslyar DJ, Heupel RC, Harada JJ (1989) Spatial patterns of gene expression in Brassica napus seedlings : identification of a cortex-specific gene and localization of mRNAs encoding isocitrate lyase and a polypeptide homologous to proteinases. The Plant Cell 1:73-80

Figdore SS, Kennard WC, Song KM, Slocum MK, Osborn TC (1988) Assessment of the degree of restriction fragment length polymorphism in Brassica. Theor Appl Genet 75:833-840

Gerlach WL, Bedbrook JR (1979) Cloning and characterization of ribosomal RNA genes from wheat and barley. Nucleic Acids Res 7:1869-1885

Gomez-Campo C, Hinata K (1980) A check list of chromosome numbers in the tribe Brassiceae. In: Tsunoda S, Hinata $\mathrm{K}$, Gomez-Campo C (eds) Brassica crops and wild allies: biology and breeding. Jpn Sci Soc Press, Tokyo, pp 51-63

Harada JJ, Baden CS, Comai L (1988) Spatially regulated genes expressed during seed germination and postgerminative devel- 
opment are activated during embryogeny. Mol Gen. Genet 212:466-473

Helentjaris T, Weber DF, Wright S (1986) Use of monosomics to map cloned DNA fragments in maize. Proc Natl Acad Sci USA 83:6035-6039

Helentjaris T, Weber D, Wright S (1988) Identification of the genomic locations of duplicate nucleotide sequences in maize by analysis of restriction fragment length polymorphisms. Genetics 118:353-363

Hosaka K, Kianian SF, McGrath JM, Quiros CF (1990) Development and chromosomal localization of genome-specific DNA markers of Brassica and the evolution of amphidiploids and $\mathrm{n}=9$ diploid species. Genome 33:131-142

McGrath JM (1989) Generation of monosomic alien addition lines in Brassica and their use in comparative evolutionary studies. Ph.D. Thesis, University of California, Davis, Calif., USA

McGrath JM, Quiros CF (1990) Generation of alien chromosome addition lines from synthetic Brassica napus: morphology, cytology, fertility and chromosome transmission. Genome, in press

Morinaga $\mathrm{T}$ (1934) On the chromosome number of Brassica juncea and $B$. napus, on the hybrid between the two, and on offspring line of the hybrid. Jpn J Genet 9:161-163

Nishi S (1980) Differentiation of Brassica crops in Asia and the breeding of 'Hakuran', a newly synthesized leafy vegetable. In: Tsunoda S, Hinata K, Gomez-Campo C (eds) Brassica crops and wild allies: biology and breeding. Jpn Sci Soc Press, Tokyo, pp 133-150

Palmer JD, Shields CR, Cohen DB, Orton TJ (1983) Chloroplast DNA evolution and the origin of amphidiploid Brassica species. Theor Appl Genet 65:181-189

Pichersky E, Bernatzky R, Tanksley SD, Cashmore AR (1986) Evidence for selection as a mechanism in the concerted evolu- tion of Lycopersicon esculentum (tomato) genes encoding the small subunit of ribulose-1,5-bisphosphate carboxylase/oxygenase. Proc Natl Acad Sci USA 83:3880-3884

Quiros CF, Ochoa O, Kianian S, Douches D (1987) Analysis of the Brassica oleracea genome by the generation of $B$. campestris-oleracea chromosome addition lines. Theor Appl Genet 74:758-766

Robbelen G (1960) Bietrage zur. Analyse des Brassica-Genomes. Chromosoma 11:205--208

Simon AE, Tenbarge KM, Scofield SR, Finkelstein RR, Crouch ML (1985) Nucleotide sequence of a cDNA clone of Brassica napus $12 \mathrm{~S}$ storage protein shows homology with legumin from Pisum sativum. Plant Mol Biol 5:191-201

Slocum MK (1989) Analyzing the genomic structure of Brassica species and subspecies using RFLP analysis. In: Helentjaris T, Burr B (eds) Development and application of molecular markers to problems in plant genetics. Cold Spring Harbor Laboratory Press, Cold Spring Harbor, New York, pp 73-80

Song KM, Osborn TC, Williams PH (1988) Brassica taxonomy based on nuclear restriction fragment length polymorphism (RFLPs). 1. Genome evolution of diploid and amphidiploid species. Theor Appl Genet 75:784-794

U, N (1935) Genomic analysis in Brassica with special reference to the experimental formation of $B$. napus and peculiar mode of fertilization. Jpn J Bot 7:389-452

Young ND, Miller JC, Tanksley SD (1987) Rapid chromosomal assignment of multiple genomic clones in tomato using primary trisomics. Nucleic Acids Res 15:9339-9348

Communicated by R.G. Herrmann 